\title{
Integrating storage in planning of LV distribution networks with PV production
}

\author{
Ahmed Hadjsaid, Marie-Cecile Alvarez-Herault, Vincent Debusschere, Raphaël Caire \\ Univ. Grenoble Alpes, CNRS, Grenoble INP*, G2Elab, 38000 Grenoble, France \\ * Institute of Engineering Univ. Grenoble Alpes \\ *E-mail: marie-cecile.alvarez@g2elab.grenoble-inp.fr, vincent.debusschere@g2elab.grenoble-inp.fr, raphael.caire@g2elab.grenoble-inp.fr
}

\begin{abstract}
This article studies the issues of storage in the planning of LV networks. An algorithm of advanced automation function is developed to illustrate the maximum economic value of storage, from the Distribution System Operator (DSO) point of view, in the long-term planning of LV networks, compared to a conventional reinforcement method. In order to model the randomness that characterizes the new actors of the network (distributed production and storage), a stochastic planning study with uncertainties concerning the connection nodes of the batteries and the photovoltaic production in the network is conducted. The results show that, compared to reinforcement, storage can present an economic benefit for the DSO, based on a limited but crucial set of elements: cost of remuneration for using storage, topology and constraints on the network, and characteristics of the equipments presenting the constraints. For the third-party storage facility owner, the presented benefits constitute the maximal envelop within which a cost benefit analysis should be restricted to be economically viable, as likely to be used to provide services to the DSO. These points are illustrated with two real networks in order to validate the proposed planning tool.
\end{abstract}

\section{Introduction}

Distribution System Operators (DSOs) are responsible for maintaining the efficient operation of distribution networks to ensure the availability and quality of supply at the lowest cost. This involves investments in components, such as lines, transformers and protections, among others, to cope with technical constraints, for example. The integration of distributed generation can increase the occurrence and intensity of constraints on networks $[1,2]$ which can be a significant financial burden, especially regarding capital expenditure (CAPEX). It is therefore essential to look for new solutions that can limit these investments over time, without degrading the operating quality or increasing costs. At the same time, the development of new sources of flexibility, such as load and source management or storage, offers new solutions to be integrated into future planning methods. The evolution of planning methods is then a challenge, considering the transition of the power system.

Several research projects have proposed alternatives to reinforcement in planning methods. For example, it is possible to optimize the load control and distributed generation to cope with voltage overshoot [3]; it is also possible to find constraint resolution methods based on load or source control and network reconfiguration [4] as well as an optimal load shedding scheme to satisfy typical constraints [5]. With the same objective, [6] analyzes the impact of the control of the assets present in the network (like local generation or storage through electric vehicles) on the resolution of technical constraints and associating business models to their usage [7].

In another domain, several publications have proposed to increase the storage to solve network constraints in the context of the development of variable renewable energy. Various studies have shown the efficiency of storage in solving voltage problems caused by a massive insertion of distributed generation [8]. [9] and [10] propose a voltage adjustment on low voltage (LV) networks, where the batteries are coordinated so as to determine the active and reactive power that must be provided or absorbed by each battery system. In [11], batteries are used for voltage regulation in LV networks. The participation of batteries for this adjustment is based either on the installed capacity of each battery or on their state of charge. [12] provides optimal power flow for voltage regulation on an LV network using storage systems.
Another possibility is to use storage as a mean to adjust consumption to production through energy prices $[13,14]$. This allows self-consumption, so less stress on the network, which improves the quality of the supply, in particular reducing voltage constraints and losses. The downside of this method is that a battery is associated with each photovoltaic $(\mathrm{PV})$ production unit, which involves a high investment cost. [15] proposes a storage management strategy to increase the PV insertion rate in distribution networks based on the minimization of a function that includes the storage cost, the losses in the network, and the voltage deviations. One of the main limitation to all these studies is that the intrinsic uncertainty of LV networks is not taken into account (for example, the location of the distributed generation and storage units).

Storage is also used for peak shaving in medium voltage (MV) networks $[16,17]$. An entire planning study of MV networks using storage is presented in [17], but no distributed generation is considered. In [16], no economic study is made, but the cost of storage poses a real problem in current long term scenarios. Optimization in the storage sizing and location is essential in LV and MV networks, as shown in [18-20]. However, the DSO may not have control over the location of the storage units, which further complicates operations.

Storage systems are usually studied from the point of view of a third party, usually cost-benefit analyses and business models [21]. In all the described studies based on storage, none has addressed the problem of the long-term planning of distribution networks by considering storage, except [17], which has addressed a MV planning problem, but without considering distributed generation. Moreover, these transformations of the electric landscape and the resulting consequences are also significant for a LV electricity network, where the aggravating factors are local, like the progression of distributed generation (in particular solar). In addition, these new players are mainly connected in single-phase, which can worsen the issue of imbalance in LV networks $[2,22]$. Studies have also been conducted to enhance conventional LV network planning methods with risk-based considerations [23]. In the end, the planning of LV networks must take into account the uncertainties of variable production as well as an increased share of local storage.

Thus, in the existing literature, the interest of storage from the DSO point of view is missing and this paper brings the following innovative contributions: 
- Development of an optimal storage management strategy enabling the DSO to avoid voltage and current constraints in his distribution network;

- Computation of the maximal cost of the storage system operation as an alternative to line upgrades in a distribution network in the case the storage is owned by a third party who rent services to the DSO;

- Integration of the uncertainties regarding the location and size of storage and PV units on the decision-investment process;

- Cost benefit analysis of the economic value of storage for the DSO in the LV network planning comparing to the business as usual planning method.

Note that, in this paper, the storage system is considered as a component providing a given service to the network, from the DSO point of view. Considering that third parties should have benefits to connect a storage facility to the network, the objective of this work is to be able to determine when storage would be interesting for the network in a planning perspective for DSO as a replacement to reinforcement. No cost-benefit analysis is thus directly provided for the storage system, as it is not the objective of the paper, but the economic consideration regarding storage owners is not put aside. The compromise this paper is looking for is when the storage is beneficial for the third-party owner within the constraints stated above for the DSO. The values proposed in this paper represent in fact the maximum benefits that could be shared by the DSO with storage third-party owners in a long-term planning perspective. In this context, we directly provide the limit in which a full cost benefice analysis of the storage (from the third-party point of view) should be contained in order to reach an agreement with the DSO.

\section{Problem statement}

The increased share of PV production in LV networks causes current and voltage violations. This requires investments (such as reinforcements) in order to enable a certain continuity of operation under good conditions. The objective is then to evaluate if taking into account the possibility of an energy storage capacity in the planning of LV power network is of interest. This possibility should avoid or at least delay the significant investment costs related to reinforcements, and eliminate the risks associated with an unexpected shift in distributed generation.

LV power networks are subject to developments by the DSO in a context where, in their majority, the local production of renewable energy and their connection nodes are random and not controllable. Thus, on the one hand it is hard to estimate all possible evolution scenarios and on the other hand, it is not possible to base conclusions on a single study scenario. As a result, a stochastic study based on the Monte Carlo method described in the flowchart of Fig. 1 has been carried out and is described in details in Section 3. Parameters that may be subject to hazards are: installed PV power ranges, PV generation location, and storage location (e.g. connection nodes) in the network. An optimization algorithm (presented in the paper) inside the Monte Carlo simulations enables to use the storage at the most of its availability. The planning study is not constructed to get the most optimal solution to a given problem, but rather to test a large range of solutions in order to assess the variety of their main characteristics (benefits, number of batteries, location of the batteries, etc.).

This work is purely conducted from the point of view of the DSO, considering energy storage as a service provided by a third party player, aware of the impact of the usage of this service on its material (aging, maintenance, etc.) as well as having already conducted its own cost benefit analysis, in order to propose the service of energy storage as described in this paper. The main hypothesis here is that, in a not-too-distant future, storage facilities will be economically viable in this context, which is currently stated in prospective studies. For instance, a reduction by up to $66 \%$ by 2030 is foreseen in [24]. This service can be used or not depending on its competitiveness compared to other tools available to the DSO in the operation and the planning of their networks.

When there appear constraints on an LV distribution network, DSO must choose the optimal solution that meets the technical needs and is economically acceptable. To address these constraints in the long run, this study compares reinforcement (the classical solution, called strategy 1 ) with storage (called strategy 2 ), which could be an alternative to reinforcement, from a planning perspective, considering a given charging and discharging strategy, for which a general overview of the algorithm is presented in Fig. 1.

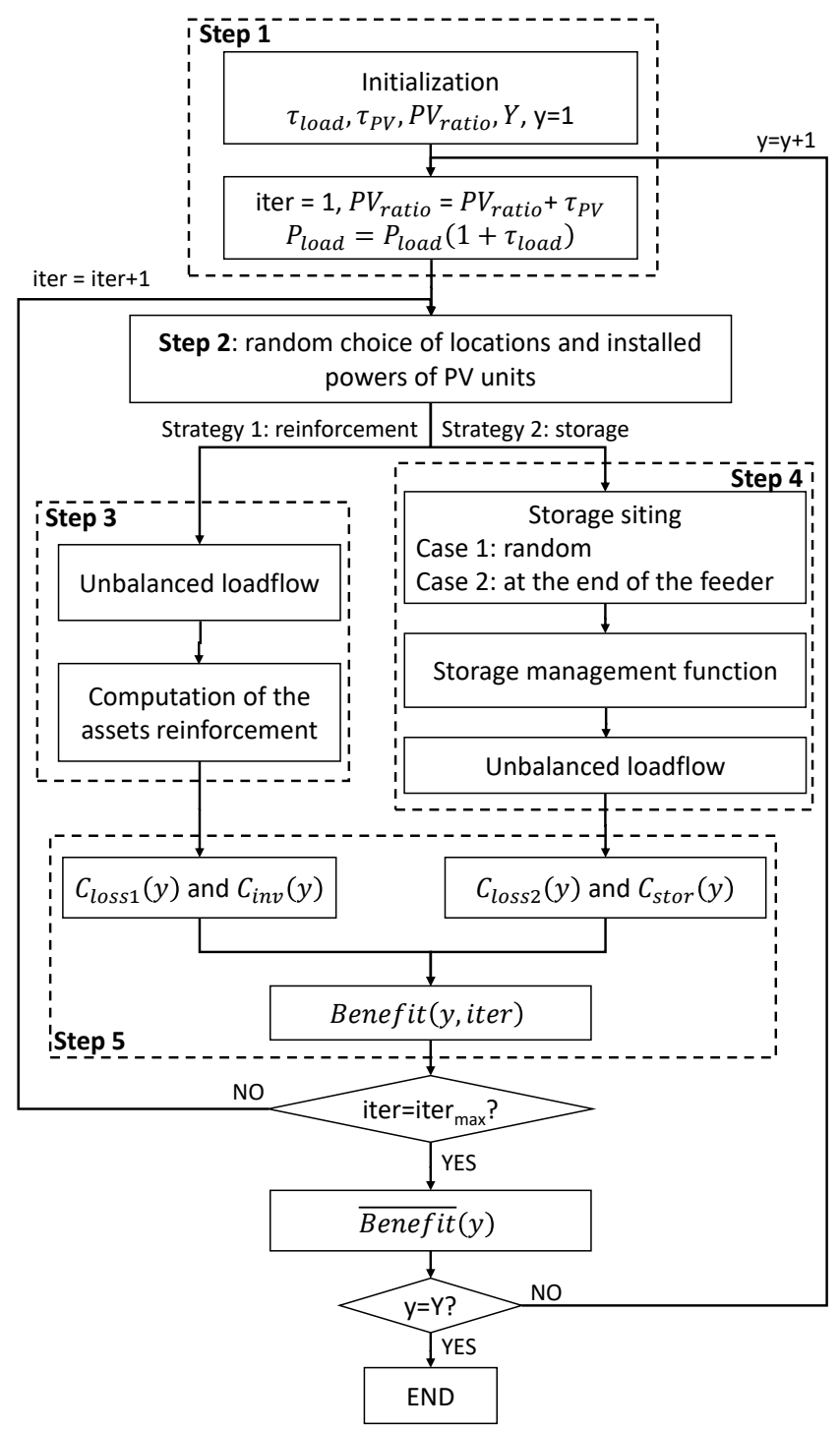

Fig. 1: General planning algorithm.

It is not possible to both reinforce and use storage in the presented planning algorithm for the sake of correctly analyzing the impact of storage systems on historical planning methods and to compare it with reinforcement from the benefit point of view. Most probably, both strategies will be applied in the real life, as the DSO may have the information on the storage capacity available.

\section{Algorithm description}

\subsection{Step 1 - Initialization}

The study is conducted on a period $Y$ of 10 years (to reduce the duration of the simulation). The peak power load increases linearly each year, following a load growth, $\tau_{\text {load }}$, taken equal to $0.9 \%$ per year. In addition, the maximum $\mathrm{PV}$ production rate, $P V_{\text {ratio }}$, defined in (1), that can be accepted by this network is estimated using a dichotomy process and is connected to the network directly at year 0 . 


$$
P V_{\text {ratio }}=\frac{\sum_{g=1}^{G} P_{\max _{\mathrm{pv}}}(g)}{\sum_{n=1}^{N} P_{\max _{\text {load }}}(n)}
$$

Where, $P_{\max _{\mathrm{PV}}}(g)$ is the installed peak power of producer $g$, $P_{\text {max load }}(n)$ is the maximum power of the load at node $n, N$ is the number of nodes in the network, and $G$ is the number of producers. The method to estimate the maximal PV production, detailed in [25], is based on Monte Carlo simulations. We can summarize this algorithm in three steps for each Monte Carlo iteration:

1. Random definition of PV production units (peak power).

2. Random distribution of PV production units in the network (choice of the connection node).

3. Unbalanced load-flow computation to estimate the state of the network [26] for the worse case (peak production and minimal consumption).

If the constraints are respected, a success is noted. Confidence intervals were computed based on the number of failures and successes obtained, leading to the convergence of the algorithm.

Several scenarios and objectives for the development of renewable energy in France exist in the literature [27, 28]. The $P V_{\text {ratio }}$ is updated each year $y$ following the NegaWatt scenario, which aims at a $100 \%$ renewable energy production for 2050 [27]. In this scenario, the share of renewable energy is equal to $17 \%$ in $2015,24 \%$ in 2020 , $55 \%$ in 2030 , and $100 \%$ in 2050 [29]. A linear increase between 2015 and 2030 is equivalent to a growth rate, $\tau_{P V}$, of $2.53 \%$ per year.

\subsection{Step 2 - random location and installed power of PV}

For both strategies 1 and 2 , the $\mathrm{PV}$ rate computed at year $y$, $P V_{\text {ratio }}(y)$, is randomly located in the LV network. The capacity of the PV units in an LV network is small compared to the facilities that can be found in MV networks [30]. In LV networks, the PV production units are often installed on the roofs of houses or just next to them (for example, small solar farms). Thus, in this study, it is only possible to connect the PV units to nodes which already have loads (consumers). Each year, and for each iteration, the installed $\mathrm{PV}$ power ranges and connection nodes are randomly selected. These random selections are based on the statistics of the installed generation in the LV networks in France [30]. The PV units are connected in single-phase if the installed power is lower than $18 \mathrm{~kW}$ peak, but three-phase otherwise [31].

\subsection{Step 3-Reinforcement strategy}

An unbalanced load-flow based on [26] is run every hour of the year and enables to estimate and locate the voltage and current constraints and then the list of lines to be reinforced in the worst case. In case of overcurrents, the reinforcement procedure consists in replacing the line under constraint by a line with a higher section. In case of voltage constraints, the feeders where the constraints appear are selected and the lines are replaced by lines with higher sections starting from the closest line to the MV/LV substation until no more constraint remains. Indeed, this method has a better impact on power losses for all the lines downstream the reinforced ones.

This load-flow also enables computing the peak power losses of the LV network and the number of hours of constraints which will be useful in section 3.5.1 to compute the cost of storage.

\subsection{Step 4 - Storage management strategy}

For the moment, the DSOs are not allowed to own flexibility assets [32]. Thus, in this paper, the storage does not belong to the DSO but rather to other actors of the electrical system of the "deregulated" domain. The DSO thus remunerates the owner of the storage for the used services. In this case, the DSO may not have control of the storage connection nodes. As the DSO is not allowed to own its storage facility, we can assume that the most likely scenario is that these storage units are closer to the producers and/or consumers. So the storage is only installed on nodes that already have loads and/or production. Two cases are considered for the installation of the storage devices. In the first case, these devices are randomly installed for each iteration of the Monte Carlo algorithm (refer to Fig. 1). In the second case, the storage devices are installed on the most constrained nodes in terms of consumption, usually at the ends of the lines. It is important to mention that the DSO does not potentially use all installed batteries, the objective being only to remove a given constraint.

3.4.1 Mathematical formulation: The storage is used during the hours when the network presents constraints (current, voltage, or maximum power of the MV/LV transformer).

Storage management is performed using an optimization function. The purpose of the optimization problem is to minimize the use of storage, knowing that there is a limit on the rated power of the lines, defined by the technical constraints. The optimization problem is formulated as a linear programming problem and solved using the optimization toolbox of Matlab. The optimization function is expressed in (2).

$$
\begin{aligned}
\min \left\{\sum_{t=1}^{T} \sum_{n=1}^{N}\left(P_{B a t, n}^{+}(t)+P_{B a t, n}^{-}(t)\right)\right. & \\
& \left.+\gamma \times \sum_{t=1}^{T} \sum_{b=1}^{B} d_{b}(t)\right\}
\end{aligned}
$$

Note that, to ensure the fact that batteries only suppress constraints, the weighting coefficient $\gamma$ should remain lower than 1. This optimization function is subject to some constraints, expressed in Equations (3)-(12), $\forall n \in\{1,2, \ldots,(N-1), N\}$, $\forall b \in\{1,2, \ldots,(B-1), B\}$ and $\forall t \in\{1,2, \ldots,(T-1), T\}$.

$$
\begin{aligned}
& S O C_{n}(t+1)=S O C_{n}(t) \\
& +P_{B a t, n}^{+}(t) \times \eta / Q_{n}^{\max } \times \Delta t \\
& -P_{B a t, n}^{-}(t) \times 1 /\left(\eta \cdot Q_{n}^{\max }\right) \times \Delta t \\
& S O C_{n}^{\min } \leq S O C_{n}(t) \leq S O C_{n}^{\max } \\
& S O C_{n}(0)=S O C_{n}(T) \\
& P_{\text {Bat, } n}^{+}>0 \\
& P_{B a t, n}^{-}>0 \\
& -P_{\text {Bat }, n}^{\min } \leq P_{B a t, n}(t) \leq P_{B a t, n}^{\max } \\
& P_{B a t, n}(t)=P_{B a t, n}^{+}(t)-P_{B a t, n}^{-}(t) \\
& \left|P_{\text {branch }, b}(t)\right| \leq P_{\text {lim }, b} \\
& d_{b}(t)=P_{\text {lim }, b}(t)-P_{\text {branch }, b}(t) \\
& {\left[\begin{array}{c}
P_{\text {branch }, 1}(t) \\
\vdots \\
P_{\text {branch }, B}(t)
\end{array}\right]=[A] \times\left[\begin{array}{c}
P_{\text {load }, 1}(t)+P_{B a t, 1}(t) \\
\vdots \\
P_{\text {load }, N}(t)+P_{B a t, N}(t)
\end{array}\right]}
\end{aligned}
$$

Where, $T$ is the number of time steps and $\Delta t$ is the considered time step. The battery power of node $n$ at the time $t$ is $P_{B a t, n}(t)$. The charge power of the battery of the node $n$ at the time $t$ is $P_{B a t, n}^{+}(t)$. The discharge power of the battery of the node $n$ at the time $t$ is $P_{B a t, n}^{-}(t)$. The minimum charge/discharge power of the battery of the node $n$ is $P_{B a t, n}^{\min }$. The maximum charge/discharge power of the battery of the node $n$ is $P_{B a t, n}^{\max }$. The difference between the power passing through branch $b$ at the time $t, P_{\text {branch }, b}(t)$, and 
the permissible power limit in this branch, $P_{l i m, N}$ is $d_{b}(t)$, with $B$ being the total number of branches of the considered network. The state of charge of the battery of the node $n$ at the time $t$ is $S O C_{n}(t)$. The initial (respectively final) state of charge of the battery of the node $n$ is $S O C_{n}(0)$ (respectively $S O C_{n}(T)$ ). The minimum state of charge of the battery of the node $n$ is $S O C_{n}^{\min }$. The maximum state of charge of the battery of the node $n$ is $S O C_{n}^{\max }$. The sum of the withdrawn and injected power at the node $n$ at $t$ is $P_{l o a d, n}(t)$. The efficiency of the battery (identical for all batteries) is $\eta$. The total capacity of the battery at the node $n$ is $Q_{n}^{\max }$. The link matrix between the powers in the branches and the powers injected in the nodes is $[A]$ [33].

3.4.2 Determination of permissible power limits: In the case where the power is limited by the maximum allowable current, (13) is used to determine $P_{l i m, b}$ in each branch.

$$
P_{l i m, b}=I_{l i m, b} \times V \times \cos \phi
$$

Where $I_{l i m, b}$ is the maximum current allowed in line $\mathrm{b}, V$ is the nominal phase/neutral voltage of a single-phase, and $\cos \phi$ is the power factor. The case where the voltage responsible for limiting the power is more complex, because the voltage on a node not only depends on the power drawn/injected at this node, but also on the consumption, production and power flow in the rest of the network to which this node belongs. The "power limits" in the network are sensitive to variations of the voltage. In the case study, the constraints are usually caused by the production. So the location of the PV producers and, more precisely, their production, are responsible for these voltage variations. Thus, to define these "power limits" as generically as possible, a Monte Carlo method was used, feeding a dichotomy algorithm.

Subsequently, to calculate the permissible power limits in each branch, we used the algorithm shown in Fig. 2. The branch $b$ (respectively $b+1)$ is considered to be the upstream line from node $n$ (respectively $(n+1)$ ). The highest PV power tested at node $n$ (upper bound) is $P_{\mathrm{PV}}^{\text {sup }}(n)$. The lowest power of PV tested at node $n$ (lower bound) is $P_{\mathrm{PV}}^{i n f}(n)$. The maximum PV power that can be connected to node $n$ without causing constraints is $P_{\mathrm{PV}}^{\max }(n)$. The limit of the $\mathrm{PV}$ production rate that is acceptable for the network is $P V_{\text {ratio }}$. The total power of $\mathrm{PV}$ acceptable for the network is $P_{\mathrm{PV}}^{l i m}$, which comes from $P V_{\text {ratio }}$ applied to (1). The stop criterion for the dichotomy algorithm is $\Delta P_{\mathrm{PV}}$.

The PV power units $\left(P_{\mathrm{PV}}^{l i m}\right)$ are randomly drawn based on the statistics of the generation installed on the LV networks in France in 2016 [30]. The connection nodes are also selected randomly according to a constant law. This step makes it possible to test several initial states for the network before calculating the power limit of the studied branch.

For a given branch, an additional power of $\mathrm{PV}, P_{\mathrm{PV}}^{\max }(n)$, is injected just at the downstream node and estimated through a dichotomy procedure as well until a situation where the line is not constrained is found. For each studied branch, $M$ iterations are carried out with a load-flow calculation to check if the constraints are respected. The considered power limit in the branch $b$ is the average of the power limits resulting from all the iterations.

It is important to mention that the initialization of $P_{\mathrm{PV}}^{\text {sup }}(n)=$ $4 \times P_{\text {load } n}$ was chosen in order to have a significantly high upper bound. This insures that the limit power of the branch $b$ is lower than the upper bound.

In the step 4, the storage management function is tested by running the unbalanced load-flow so as to compute the peak power losses and the cost of storage.

\subsection{Step 5 - Costs modeling}

In order to arbitrate between the different possible solutions in a network planning study, the DSO uses a discount principle where all expenses are computed at the reference year of the planning period by taking into account the present discounted value. In other words, a one-year investment deferral that should cost $C$ makes it possible to make a profit of $C \times i /(1+i)$ [23] the following year, where $i$ is

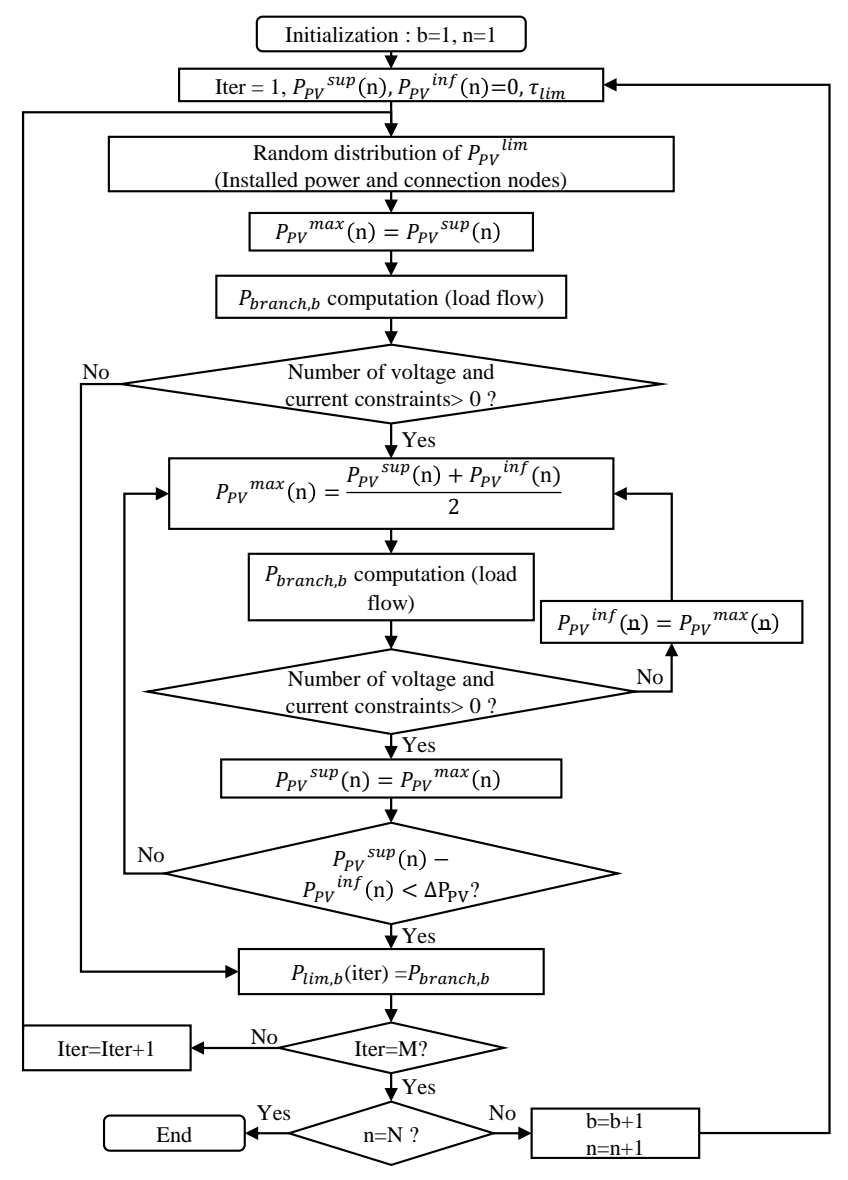

Fig. 2: Algorithm to compute the power limit of each conductor.

the discount rate, equal to $8 \%$ in this study [6]. Thus, to compute the updated total cost of each strategy (reinforcement or storage), (14) is used [23].

$$
\begin{aligned}
& C_{d i s c}=\frac{\sum_{y=1}^{Y} \operatorname{CAPEX}(y)+\operatorname{OPEX}(y)}{(1+i)^{y}} \\
& \text { with } \operatorname{OPEX}(y)= \begin{cases}C_{\text {loss }_{S 1}}(y) & \text { if strategy 1 } \\
C_{\text {loss }_{S 2}}(y)+C_{\text {stor }}(y) & \text { if strategy 2 }\end{cases} \\
& \text { and } \operatorname{CAPEX}(y)=C_{\text {inv }}(y)
\end{aligned}
$$

Where, $C_{d i s c}$ is the discounted cost of the strategy at the reference year (in $\mathrm{k} €$ ); $C_{\text {inv }}(y)$ is the amount of investment to be made in year $y$ (in $\mathrm{k} €$ ); $C_{\operatorname{loss}_{S 1}}(y)$ and $C_{\text {loss }_{S 2}}(y)$ are the costs of power losses for year $y$ (in $\mathrm{k} €$ ) for both strategies; $C_{\text {stor }}(y)$ is the cost of storing energy during year $y$ (in $\mathrm{k} €$ ) and $Y$ is the year of evaluation.

In this paper, the CAPEX is the reinforcement cost of the lines, which consists of the cost of the trenches (about $100 \mathrm{k} € / \mathrm{km}$ [29]) and the cost of the cables (from $20 \mathrm{k} € / \mathrm{km}$ to $40 \mathrm{k} € / \mathrm{km}$ depending on the section). The OPEX is the cost of the power losses and the cost of the storage (when used to remove technical constraints).

The storage strategy (strategy 2) will be more interesting than the reinforcement strategy (strategy 1) if (15) is verified.

$$
\frac{C_{l o s s_{S 1}}(y)-C_{l_{\text {oss }} \text { S2 }}(y)-C_{\text {stor }}(y)}{C_{i n v}(y)}>\frac{i}{1+i}
$$

Where, $C_{\text {loss }_{S 1}}(y)$ is the cost of the losses for strategy 1 at year $y$ and $C_{\text {loss }_{S 2}}(y)$ is the cost of the losses for strategy 2 at year $y$. From (15), a comparison criterion can be defined as the potential discounted benefit that the storage could generate, provided in (16). 


$$
\text { Benefit }(y)=\frac{C_{i n v}(y) \cdot i}{(i+1)^{(y+1)}}-\frac{C_{\text {stor }}(y)-\Delta C_{\text {loss }}(y)}{(i+1)^{y}}
$$

Where, Benefit $(y)$ is the discounted annual profit of the storage solution and $\Delta C_{\text {loss }}(y)$ is the difference between the cost of the losses for the reinforcement strategy and for the storage strategy. Consequently, if Benefit (y) is lower than zero, then at year $y$ the reinforcement is made.

3.5.1 Power losses cost computation $\left(C_{l o s s 1}, C_{l o s s 2}\right)$ : The method used by the French DSO for the computation of the annual power losses cost is given in (17) [34].

In step 3 and 4, the peak power losses of the four wire energy system are estimated to get $P_{\text {loss }}^{\max }$ as used in (17).

$$
C_{\text {loss }}=C_{1 k W P L}\left(H_{\max }\right) \times P_{\text {loss }}^{\max }
$$

Where, $C_{1 k W P L}$ is the cost of one $\mathrm{kW}$ of peak power losses $(\mathrm{k} \in / \mathrm{kW})$ given in [34]; $C_{\text {loss }}$ the annual cost of power losses $(\mathrm{k} €) ; P_{\text {loss }}^{\max }$ the maximal power losses $(\mathrm{kW}) ; H_{\max }$ the equivalent duration so that $H_{\max } \times P_{\max }$ is equal to the annual energy consumed.

3.5.2 Cost of using storage facilities $\left(C_{\text {stor }}\right)$ : Currently, storage is not used in the distribution network to provide services to the DSO. Then no economic model exists whether the storage is owned by the DSO or not. In order to make a cost benefit analysis between reinforcement and storage, to defer or avoid network investment, a model of the cost of using storage facilities by the DSO is required. The value of this cost may be inflated by:

- the type of electrical device to reinforce (transformer, cable type and length);

- the storage capacity which depends on the load and production growth rates, and consequently on the evolution of the constraints, their year of appearance and their number of occurrences each year; - the shape of the load curve.

The maximal storage cost is defined at the limit where it is just more economical than reinforcement. It is expressed in $\mathrm{k} € / \mathrm{kWh} /$ year and obtained by solving $(18)=0$.

$$
C_{\text {lim }}(y)=\frac{C_{i n v} \times i /(1+i)^{y}+\Delta C_{l o s s}(y)}{\text { Capa }_{\text {stor }}(y)}
$$

Where, $C_{\text {lim }}(y)$ is the maximal cost of storage at the year $y$ and $\operatorname{Capa}_{\text {stor }}(y)$ is the storage capacity used in year $y$.

A statistical study was performed so as to estimate the maximal cost of storage, taking into account the occurrences of a period where the constraints are violated in a given distribution network. For 4000 daily load curves and 6 different rates of load growths, load-flows have been run to identify the constraints violations and the cost of reinforcement for the network as a whole.

After estimating the storage capacity needed for removing the constraints, the maximal cost of storage is estimated. A sensitivity analysis of the type of constraints violated was run (either on lines of several lengths and on the transformer). In [35], distribution networks are classified regarding the frequency of constraints appearances. A network is weakly constrained if the duration of constraints does not exceed 50 non-consecutive hours per year or is lower than 10 consecutive hours per year. The network is highly constrained if the duration of constraints exceeds 500 non-consecutive hours per year or 20 consecutive hours per year. Between these values, the network is considered moderately constrained. Based on all those simulations, the average cost is computed for these three groups and proposed in Table 1.

These costs have been used in the case studies to validate the proposed method. The unbalanced load-flow of Step 3 provides the number of hours of constraints of the network per year which enables to select the correct costs of the storage to use in Table 1. It is nevertheless notable that the variation in these costs is quite high.
Table 1 Limit average cost of remuneration of $1 \mathrm{kWh}$ of storage.

\begin{tabular}{ll}
\hline Type & Mean cost, $€ / \mathrm{kWh}$ \\
\hline Weakly constrained & 4620 \\
Moderately constrained & 75.2 \\
Highly constrained & 3.84 \\
\hline
\end{tabular}

\section{Case studies}

The method proposed in this paper has been applied to two real LV networks presented in Fig. 3 and Fig. 6. The differences regarding the main characteristics of those networks (proposed in Table 2) justifies the comparison of the results of the planning method from the point of view of the impact of the storage systems.

\subsection{First test case}

The method proposed in this paper has been applied to the real LV network presented in Fig. 3. This network is a three-phase network with the neutral distributed and grounded. The MV/LV transformer has a nominal power of $50 \mathrm{kVA}$ and supplies 10 customers. Six of them are connected in three-phase and the other four are connected in single-phase. This network consists of 12 nodes, 6 of which have no load. The total length of the network is $2217 \mathrm{~m}$ and the cables used are aluminum, with sections of $70 \mathrm{~mm}^{2}$ and $35 \mathrm{~mm}^{2}$.

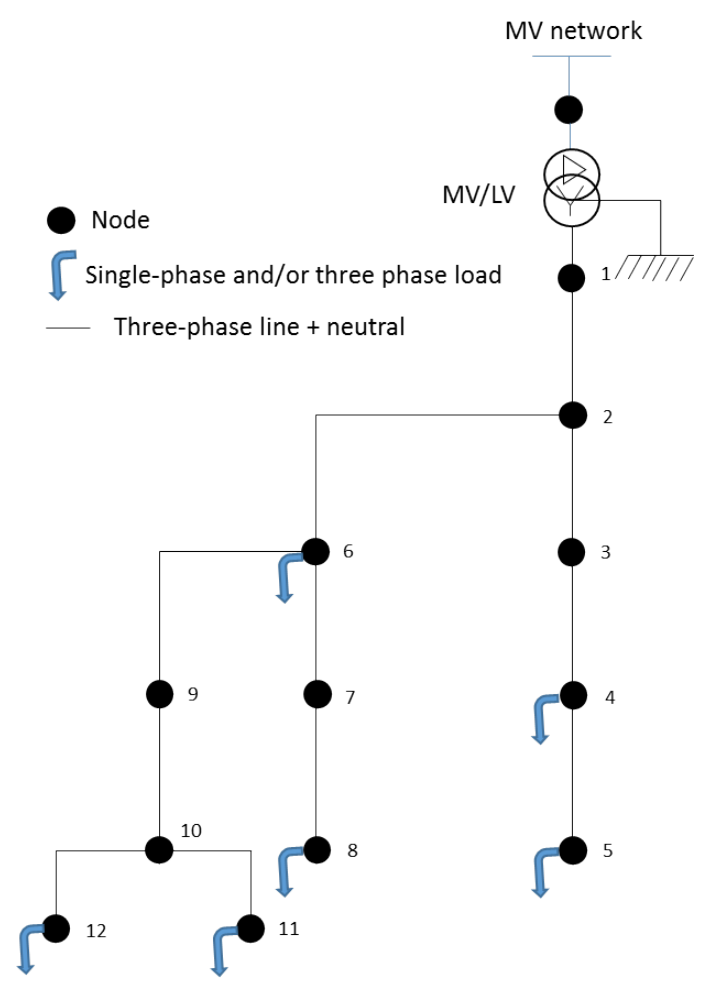

Fig. 3: LV distribution network (network 1).

The maximum amount of PV that can be connected without causing technical constraints is computed with the method described in Section 3.1 and is $21.7 \%$. It is assumed that $21.7 \%$ of the PV production is connected to the network 1 at year 0 and then increases by $2.53 \%$ each year. At the end of the study period, network 1 will have $47.5 \%$ of PV integration.

If reinforcement only is considered (strategy 1), in this case study $1650 \mathrm{~m}$ of lines are required. The corresponding investment cost is then equal to $187 \mathrm{k} €$ and is anticipated at year 1 .

The next step is to compare the global discounted cost of strategy 1 to the cost of using storage, with two scenarios: either the storage 
is randomly distributed in the network, or the location is "controlled" by the DSO.

4.1.1 Storage randomly allocated (case 1): First, for each Monte Carlo iteration, the location of the storage is randomly chosen. The LV network has 6 nodes with a non-zero consumption, three scenarios are tested $(4,5$ or 6 available batteries). Only the case with storage at every consuming node (6 batteries) enables to solve all the constraints due to the PV penetration. When the storage is located downstream the constraint, it manages to solve it. But if it is upstream or very far from the constraint, it is useless.

The benefit of storage compared to the reinforcement is computed using (16). The cost of the remuneration of the storage is linked to the state of the network, i.e. weakly, moderately, or highly constrained. In the case of network 1, the maximal number of constraints is recorded at year 7 and its average value over the Monte Carlo iterations is 626 .

It can be concluded that this network is highly constrained [35] and then the cost of remuneration will be $3.48 € / \mathrm{kWh}$ (refer to Table 1 ). As this cost may vary, a sensitivity analysis of $\pm 30 \%$ around this value has been tested, leading to assuming a remuneration cost between $2.44 € / \mathrm{kWh}$ and $4.53 € / \mathrm{kWh}$. Fig. 4 illustrates the average values of the benefit of storage over all the Monte Carlo simulations for the case of 6 batteries and the 3 remuneration costs.

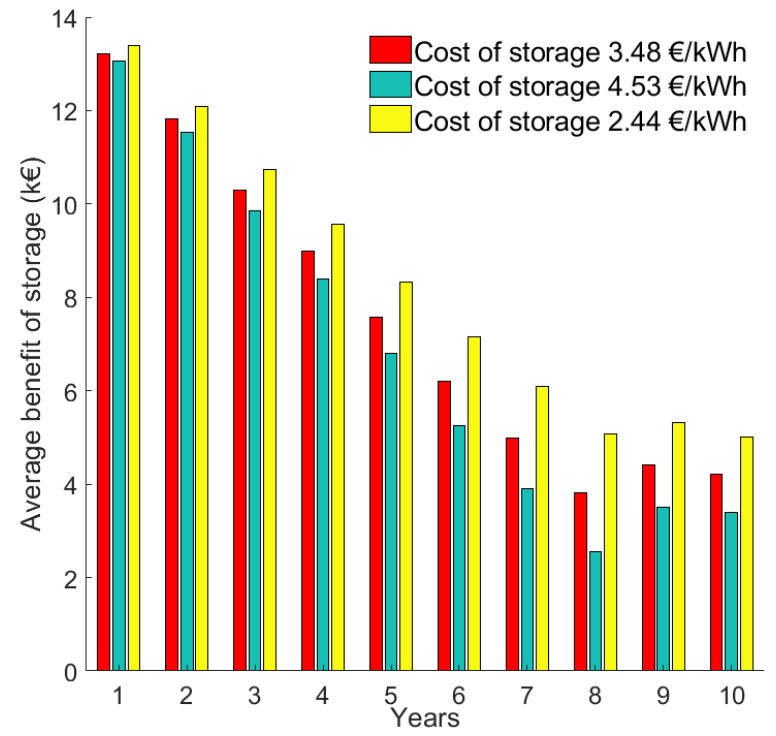

Fig. 4: Sensitivity of the average benefit to the remuneration cost of storage (case of 6 batteries).

It can be seen in Fig. 4 that storage is more economical than reinforcement in this case. The benefit is positive over the entire planning period, and varies from $3.82 \mathrm{k} €$ to $13.3 \mathrm{k} €$ depending on the remuneration cost of storage. It can be seen that the less the storage is used, the greater the benefit will be. Consequently, the impact of the remuneration cost of storage increases with the number of constraints. For example, at year 8 , reducing this cost by $30 \%$ increases the benefit by $33 \%$. In addition, at year 0 , the $\mathrm{PV}$ penetration is the lowest and so is the number of constraints. As the storage is not used a lot, its benefit is high and a variation of $30 \%$ of the remuneration cost makes a significant variation of the benefit.

Note that the benefit is maximal at the beginning of the studied period and then decreases. Indeed, in the case of reinforcement, the investment is done only in year 0 , because the DSO plans ahead so as to minimize the power losses of the network. Then, year by year, more constraints appear, due to the increase of PV production. In the case of reinforcement, no additional investment is required, but in the case of storage, as more and more constraints appear, the required capacity of storage increases too.
Nevertheless, after the $9^{\text {th }}$ year, the benefit increases smoothly. This is due to the PV penetration, which becomes very high and consequently in the Monte Carlo algorithm there is an increased probability that a three-phase connection is chosen rather than a single-phase connection. This phenomenon tends to balance the network and so reduce the constraints.

4.1.2 Imposed storage location (case 2): In the case where the location of storage can be chosen, two scenarios have been considered: three or four batteries are located at the end of the lines where the constraints are maximal. Only in the case of four batteries are all the constraints respected at the end of the planning study. The results are depicted in Fig. 5. The scenarios with 6 or 4 batteries are compared, so as to quantify the importance of minimizing their number.

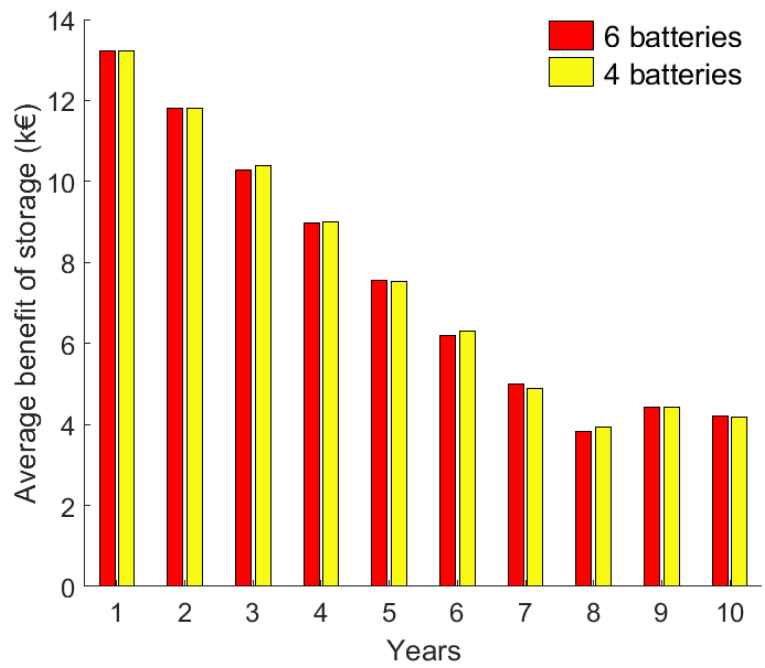

Fig. 5: Random location of batteries versus batteries at the end of the network.

Only one remuneration cost is shown $(3.84 € / \mathrm{kWh})$. The same tendency and explanations can be made for the other remuneration costs. Indeed, the costs are almost the same, and the difference is mainly due to power losses. The power required to remove the constraint will be dependent on the path taken by the energy flows.

4.1.3 Third-party storage facility owner: For the storage facility owner, acting as a third-party player, these results directly present the maximal envelop within which a cost benefit analysis should be contained if the storage facility owner is willing to propose financialy attractive services to the DSO. Current CAPEX and OPEX of storage facilities in the literature vary significantly per $\mathrm{kWh}$ of installed capacity, depending on the voltage level, the technology, the installed capacity, and the associated usage [36, 37]. Therefore it is much more realistic to just describe those results as limits within which a storage owner should contain its cost benefit analysis. Nevertheless, authors in [38] proposed an algorithm to compute the levelized cost of energy (LCOE) of various storage technologies. Considering the ones which can be used at the distribution level, the LCOE varies from $0.03 € / \mathrm{kWh}$ and $0.27 € / \mathrm{kWh}$ which is 12 to 116 times lower than the remuneration cost considered in this paper. In this context, the economic viability of storage facilities are within reach for this study case.

\subsection{Second test case}

Simulations were run on another type of LV network. The topology of the network is presented in Fig. 6.

Table 2 compares networks 1 and 2 . The initial PV penetration is $42.9 \%$ (year 0 ) and $68.2 \%$ at the end of the planning period. The results also show that it is necessary to have one battery in every 


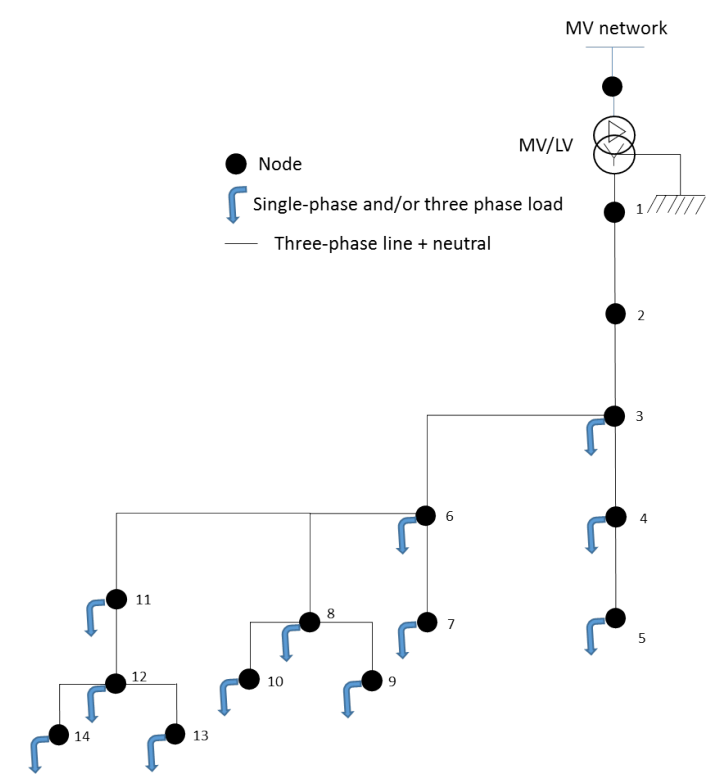

Fig. 6: LV distribution network (network 2).

consumption nodes (in the case of random location). In the case of batteries located at the end of the lines, 6 of them are required. The average number of constraints recorded in the last year is 1500 , so it is a highly constrained network [35].

Table 2 Comparison of network 1 and 2.

\begin{tabular}{lll}
\hline Characteristic & Network 1 & Network 2 \\
\hline MV/LV transformer (kVA) & 50 & 250 \\
Number of customers & 10 & 23 \\
Three-phase customers & 6 & 11 \\
Single-phase customers & 4 & 12 \\
Number of nodes & 12 & 14 \\
Number of consuming nodes & 6 & 12 \\
Total length (m) & 2220 & 810 \\
Section used (mm $\left.{ }^{2}\right)$ & $35 ; 70$ & $35 ; 70 ; 150$ \\
Maximum consumption $(\mathrm{kW})$ & 42 & 182 \\
\hline
\end{tabular}

Fig. 7 shows the average benefit for the second network. In this case, the benefit of storage is negative, meaning that the reinforcement is more relevant than the storage. Indeed, network 2 has a higher load density and shorter lines than network 1 . In that case, there is no economic viability for a third-party storage owner.

Consequently, the total length of the lines to be reinforced for network 2 is $810 \mathrm{~m}$ (about $36,5 \%$ of the network) involving a cost of about $93 \mathrm{k} €$, which is roughly half that of network 1 . Incidentally, in the case of network 2, the load density, the PV penetration, and the number of nodes are significant, making the need for storage increase as well as its cost of remuneration.

At the end of the planning period, the number of constraints for network 2 is 2.5 times higher than for network 1 .

If the DSO does not own the batteries and does not choose their location, the storage enables differing investments, while taking into account the uncertainties linked to production and consumption, only if it is available in all the nodes where consumption and production are available. Nevertheless, if incentives exist, so as to control the location of batteries, then storage can be a good alternative to reinforcement if it is located at the end of the lines of the network.

The characteristics of the network can also have an impact on the advantageousness of storage. For a network with long lines and a high total conductor length to be reinforced, it will be more economical to use storage because the investment will be high. For a network having low investment needs or a high need for storage, then the reinforcement may be more economical.

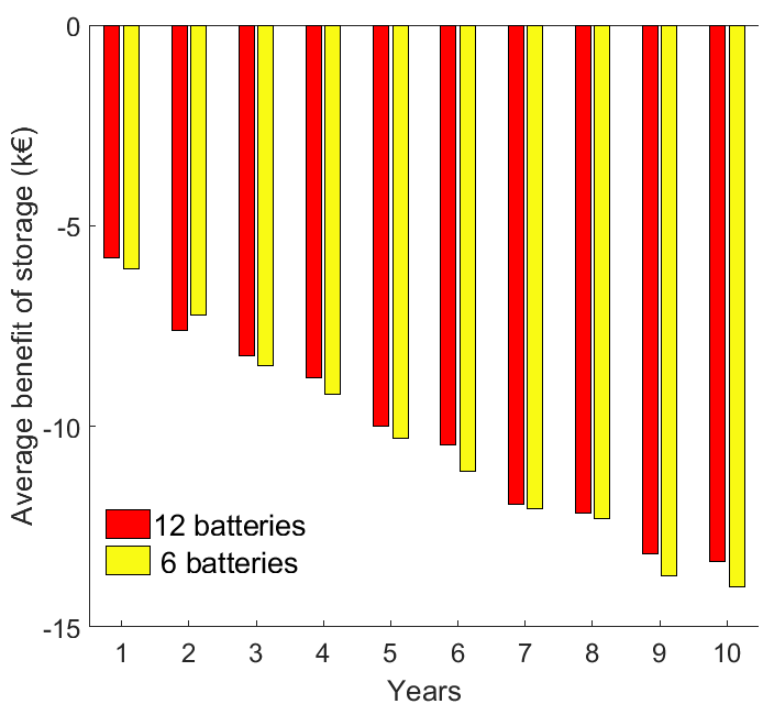

Fig. 7: Average benefit of storage for network 2 (in red, random location of 12 batteries, in yellow, 6 batteries located at the end of the lines).

\section{Conclusion}

In this paper, a technical and economic analysis of the use of storage as an alternative to reinforcement in a LV distribution network is proposed, including an optimization to model the operation of storage so as to relieve network voltage or current constraints. This algorithm has been integrated with a stochastic simulation based on the Monte Carlo method so as to model the uncertainties of the load and production. This method has been applied to two different real LV networks in order to show situations where storage is more economic than reinforcement and others where the contrary is observed. Considering only the DSO point of view, the main conclusion is that the benefits of storage depend on:

- the cost of remuneration of storage;

- the evolution of the constraints during the study period (the more constraints appear, the lower the benefits will be);

- the characteristics of the equipment presenting a constraint (for example, the longer the line to be reinforced, the higher the cost of investment will be, and the more economical the storage will be).

This study also shows the challenges associated with the knowledge of the capacity and the location of the storage in the network. In a context where the DSO does not necessarily manage the location of storage, it can be concluded that the storage can differ or avoid a reinforcement only if all the consumers/producers nodes are equipped with storage system.

For the third-party storage facility owner, these results directly present the maximal envelop within which a cost benefit analysis should remain if ever willing to propose services to the DSO. A preliminary comparison with currents CAPEX and OPEX of storage facilities shows that economic viability of storage facilities is within reach only for one study case compared to reinforcement.

Finally, this paper focuses on the case where the DSO is not allowed to own a storage system but can rent its services. A further study would be to investigate the case where the DSO can own the storage units and then would need to find the optimal location and sizing of storage. This problem would be dramatically different in terms of mathematical formulation and algorithm development.

\section{References}

1 D. Santos-Martin and S. Lemon, "Simplified modeling of low voltage distribution networks for PV voltage impact studies," IEEE Transactions on Smart Grid, vol. 7 , no. 4, pp. 1924-1931, Jul. 2016. 
2 P. K. C. Wong, A. Kalam, and R. Barr, "Modelling and analysis of practical options to improve the hosting capacity of low voltage networks for embedded photovoltaic generation," IET Renewable Power Generation, vol. 11, no. 5, pp. 625-632, 2017.

3 E. Belič, N. Lukač, K. Deželak, B. Žalik, and G. Štumberger, "GPU-Based online optimization of low voltage distribution network operation," IEEE Transactions on Smart Grid, vol. 8, no. 3, pp. 1460-1468, May 2017.

4 A. Kulmala, M. Alonso, S. Repo, H. Amaris, A. Moreno, J. Mehmedalic, and Z. Al-Jassim, "Hierarchical and distributed control concept for distribution network congestion management," IET Generation, Transmission \& Distribution, vol. 11 , no. 3, pp. 665-675, 2017.

5 V. Gouin, M.-C. Alvarez-Hérault, and B. Raison, "Optimal planning of urban distribution network considering its topology," in 23th Int. Conf. on Electricity Distribution. CIRED, Jun. 2015.

6 B. D. Tavares, J. Sumaili, F. J. Soares, A. G. Madureira, and R. Ferreira, "Assessing the impact of demand flexibility on distribution network operation," in PowerTech. Manchester, UK: IEEE, Jun. 2017, pp. 1-6.

7 N. Günter and A. Marinopoulos, "Energy storage for grid services and applications: classification, market review, metrics, and methodology for evaluation of deployment cases," Journal of Energy Storage, vol. 8, pp. 226-234, 2016.

8 M. Zidar, P. S. Georgilakis, N. D. Hatziargyriou, T. Capuder, and D. Škrlec, "Review of energy storage allocation in power distribution networks: applications, methods and future research," IET Generation, Transmission \& Distribution, vol. 10 , no. 3, pp. 645-652, 2016

9 Y. Wang, B. F. Wang, and P. L. So, "A voltage regulation method using distributed energy storage systems in LV distribution networks," in Int. Energy Conf. IEEE, Apr. 2016, pp. 1-6.

10 Y. Wang, K. T. Tan, X. Y. Peng, and P. L. So, "Coordinated control of distributed energy storage systems for voltage regulation in distribution networks," IEEE Transactions on Power Delivery, vol. 31, no. 3, pp. 1132-1141, Jun. 2016.

11 M. Zeraati, M. E. H. Golshan, and J. Guerrero, "Distributed control of battery energy storage systems for voltage regulation in distribution networks with high PV penetration," IEEE Transactions on Smart Grid, vol. PP, no. 99, p. 1, 2016.

12 H. M. Costa, J. Sumaili, A. G. Madureira, and C. Gouveia, "A multi-temporal optimal power flow for managing storage and demand flexibility in LV networks," in PowerTech. Manchester, UK: IEEE, Jun. 2017, pp. 1-6.

13 F. Lamberti, V. Calderaro, V. Galdi, and G. Graditi, "Massive data analysis to assess PV/ESS integration in residential unbalanced LV networks to support voltage profiles," Electric Power Systems Research, vol. 143, pp. 206-214, 2017.

14 F. Bignucolo, A. Savio, R. Turri, N. Pesavento, and M. Coppo, "Influence of electricity pricing models on the daily optimization of residential end-users integrating storage systems," in Modern Power Systems (MPS), Jun. 2017, pp. 1-6.

15 N. Jayasekara, P. Wolfs, and M. A. S. Masoum, "An optimal management strategy for distributed storages in distribution networks with high penetrations of PV," Electric Power Systems Research, vol. 116, pp. 147-157, 2014.

16 M. Rowe, T. Yunusov, S. Haben, C. Singleton, W. Holderbaum, and B. Potter, "A peak reduction scheduling algorithm for storage devices on the low voltage network," IEEE Transactions on Smart Grid, vol. 5, no. 4, pp. 2115-2124, Jul. 2014

17 X. Shen, M. Shahidehpour, Y. Han, S. Zhu, and J. Zheng, "Expansion planning of active distribution networks with centralized and distributed energy storage systems," IEEE Transactions on Sustainable Energy, vol. 8, no. 1, pp. 126-134, Jan. 2017.

18 S. Hashemi, J. Østergaard, and G. Yang, "A scenario-based approach for energy storage capacity determination in LV grids with high PV penetration," IEEE Transactions on Smart Grid, vol. 5, no. 3, pp. 1514-1522, May 2014.

19 A. Giannitrapani, S. Paoletti, A. Vicino, and D. Zarrilli, "Optimal allocation of energy storage systems for voltage control in LV distribution networks," IEEE Transactions on Smart Grid, vol. 8, no. 6, pp. 2859-2870, Nov. 2017.

20 M. Zidar, P. S. Georgilakis, N. D. Hatziargyriou, T. Capuder, and D. Škrlec, "Review of energy storage allocation in power distribution networks: applications, methods and future research," IET Generation, Transmission \& Distribution, vol. 10, no. 3, pp. 645-652, 2016 .

21 B. Hartmann, D. Divényi, and I. Vokony, "Evaluation of business possibilities of energy storage at commercial and industrial consumers - a case study," Applied Energy, vol. 222, pp. 59-66, 2018

22 M. J. E. Alam, K. M. Muttaqi, and D. Sutanto, "Alleviation of neutral-to-ground potential rise under unbalanced allocation of rooftop PV using distributed energy storage," IEEE Transactions on Sustainable Energy, vol. 6, no. 3, pp. 889-898, Jul. 2015 .

23 M. Nijhuis, M. Gibescu, and S. Cobben, "Risk-based framework for the planning of low-voltage networks incorporating severe uncertainty," IET Generation, Transmission \& Distribution, vol. 11, no. 2, pp. 419-426, 2017.

24 P. Ralon, M. Taylor, A. Ilas, H. Diaz-Bone, and K.-P. Kairies, "Electricity storage and renewables: costs and markets to 2030" International renewable energy agency (IRENA), techreport, Jul. 2016, [Accessed: March-2019]. [Online]. Available: https://www.irena.org/-/media/Files/IRENA/Agency/Publication/2017/ Oct/IRENA_Electricity_Storage_Costs_2017.pdf

25 M. Alvarez-Herault, A. Battegay, C. Gandioli, J. Descloux, N. Hadjsaid, and P. Tixador, "Maximal DG indicator to quantify the efficiency of smart grid solutions regarding renewable penetration," in PowerTech. Grenoble, France: IEEE, Jun. 2013, pp. 1-5.

26 R. M. Ciric, A. P. Feltrin, and L. F. Ochoa, "Power flow in four-wire distribution networks-general approach," IEEE Transactions on Power Systems, vol. 18, no. 4, pp. 1283-1290, 2003.

27 Greenpeace, EREC, and GWEC, "Scénario de transition énergétique (energy transition scenario), (in French)," Greenpeace France, techreport, 2013, [Accessed: March-2019]. [Online]. Available: https://cdn.greenpeace.fr/site/uploads/2017/02/ Scenario-Transition-Energetique-Greenpeace-2013.pdf
28 J.-F. Raux, "Electricité 2030: quels choix pour la France? (Electricity 2030: what choices for France?), (in French)," Revue de l'Energie, vol. 1, no. 605, 2012.

29 V. Gouin, M. Alvarez-Hérault, and B. Raison, "Benefits of load shedding for distribution grids investments planning," in Power \& Energy Society General Meeting (PES GM). IEEE, 2015, pp. 1-5.

30 Enedis, "Parc des installations de production raccordées sur le réseau Enedis par tranche de puissance (Park of production facilities connected to the Enedis network per power unit), (in French)," Enedis, techreport, 2019, [Accessed: March-2019]. [Online]. Available: https://data.enedis.fr/explore/dataset/ parc-des-installations-de-production-raccordees-par-departement/information/

$31 \stackrel{\text {, }}{2}$, Barème pour la facturation des raccordements au réseau public de distribution d'électricité concédé à Enedis (Billing of the connections to the public electricity distribution network granted to Enedis), (in French)," Enedis, techreport, Jul. 2018, [Accessed: March-2019]. [Online]. Available: https://www.enedis.fr/sites/default/files/Enedis-PRO-RAC_03E.pdf

32 N. Chabanne and A. Schneider, "Rapport d'information déposé par la commission des affaires européennes sur la nouvelle organisation du marché de l'électricité, dans le cadre du quatrième paquet de l'énergie (Information report tabled by the European Affairs Committee on the new organization of the electricity market, as part of the fourth energy package), (in French)," European Affairs Committee, techreport, Mar. 2017, [Accessed: March-2019]. [Online]. Available: http://www.assemblee-nationale.fr/14/europe/rap-info/i4568.asp

33 J.-H. Teng, "A direct approach for distribution system load flow solutions," IEEE Transactions on Power Delivery, vol. 18, no. 3, pp. 882-887, Jul. 2003.

34 A. Hadjsaid, M.-C. Alvarez-Herault, V. Debusschere, and R. Caire, "Integration of storage and pv in the dso power losses cost assessment method for lv planning studies," in 25th International Conference on Electricity Distribution (CIRED), Madrid, Spain, Jun. 2019, pp. 1-5.

35 E-CUBE Strategy Consultants, "Etude sur la valeur des flexibilités pour la gestion et le dimensionnement des réseaux de distribution (Study on the value of flexibilities for the management and sizing of distribution networks), (in French)," Commission de régulation de l'énergie (CRE), techreport, Jul. 2017. [Online]. Available: https://www.cre.fr/content/download/14046/168032

36 B. Idlbi, J. von Appen, T. Kneiske, and M. Braun, "Cost-benefit analysis of battery storage system for voltage compliance in distribution grids with high distributed generation," Elsevier Energy Procedia, vol. 99, pp. 215-228, 2016.

37 C. Mateo, J. Reneses, A. Rodriguez-Calvo, P. Frías, and . Sánchez, "Costbenefit analysis of battery storage in medium-voltage distribution networks," IET Generation, Transmission \& Distribution, vol. 10, no. 3, pp. 815-821, 2016.

38 M. Obi, S. M. Jensen, J. B. Ferris, and R. B. Bass, "Calculation of levelized costs of electricity for various electrical energy storage systems," Renewable and Sustainable Energy Reviews, vol. 67, pp. 908-920, 2017. 